\title{
Finding Optimal Configuration of DSDV using Particle Swarm Optimization
}

\author{
Sanjiv Sharma \\ Shobhit University \\ Meerut, India
}

\author{
A. K. Giri \\ KIET Group of Institution \\ Ghaziabad, India
}

\author{
Niraj Singhal, PhD \\ Shobhit University \\ Meerut, India
}

\begin{abstract}
Vehicular Ad-hoc network (VANET) is prominent research area in Mobile ad-hoc network (MANET). VANET are very dynamic in nature as it has no predefined structure for communication. The performance of VANET dependents on parameter configuration of the protocol used in. The optimal parameter configuration in protocol can improve the QoS of VANET. Further, finding the optimal values of the parameters configuration is not easy because there is multiple combination of parameters configuration. Therefore, we have used particle swarm optimization technique, a metaheuristic, to find the optimal parameter configuration in real scenario. The result of experiment shows that there is $11.96 \%$ drop in average End to End Delay, 5.42\% drop in Normalized Routing load and $5.74 \%$ gain in Packet Delivery based on the optimal configuration found against the default parameter configuration in DSDV.
\end{abstract}

\section{Keywords}

PSO, NS-2, DSDV.

\section{INTRODUCTION}

Vehicular Ad hoc Network (VANET) is one of the branches of MANET which requires no pre existing infrastructure and has dynamic nature in communication. This dynamic nature is because of its topology changing behavior. The topology changes very quickly because the movement of vehicles follows the road constraints and the range of communication of the vehicles are limited [1]. In such volatile network, it is difficult to achieve the good QoS of VANET in real scenario.

The QoS depends on the parameters configuration of protocol being used in VANET for routing of messages. The parameter configuration, which may be different for different scenario, affects the QoS. The possible combinations of parameters configuration are huge and it is very difficult to find optimal parameters configuration for VANET which can improve the QoS of VANET. This motivates us to use metaheuristic [2] to find the optimal parameters configuration. Metaheuristic is used to find the optimal value from search space which has nature of combinatorial optimization problems. In our problem, we have taken particle swarm optimization technique.

There are very few applications of metaheuristic in VANET. E. Alba [3] has used Genetic Algorithm to find the optimal broadcasting strategy in MANET. Vanhatupa el. Al [4] has used flexible Genetic Algorithm for optimization of channel assignment in mesh wireless network. Di Caro [5] used Ant Colony Optimization for implementing new routing protocols for MANETs. Garcia-Nieto [6] has used five metaheuristic techniques Particle Swarm Optimization, Differential
Evolution, Genetic Algorithm, Evolution Strategy and Simulated Annealing algorithms for finding of optimal set of configuration parameter's value to tune AODV automatically. Recently Huang [7] has proposed Particle Swarm optimization based new routing protocol to reduce packet loss rate in theoretical VANET scenario.

In this paper, we have tried to find out optimal parameters configuration of DSDV protocol using Particle Swarm Optimization (PSO). PSO is a population based stochastic optimization technique, inspired by social behavior of bird flocking or fish schooling. We have chosen this algorithm because of its suitability to our problem. The network simulator NS-2 [8] is used for evaluation of parameters configuration generated by PSO. PSO is guided by the fitness function which is formed based on ns-2 trace results. The real map of Ghaziabad UP India, has been taken so that evaluation of parameters configuration be realistic. Our experiments show that the produced parameters configuration of DSDV improves the QoS of VANET as compared to the default parameters configuration of DSDV.

The remaining of this paper is organized as follows. In section 2, DSDV protocol is introduced with its parameters. Section 3 consists of a framework of optimization strategy. Section 4 describes VANET scenario and mobility model being used. Experimental setup, mapping of problem into PSO, comparisons and result analysis are discussed in section 5 . Finally section 6 , discusses the conclusion and future work which can be carried out in this field.

\section{DSDV}

DSDV is proactive and sometime it is called table driven. The working of DSDV is based on Bellman Ford algorithm. This is developed by Charles, E Perkins and Pravin Bhagwal in 1994 [8]. In DSDV, a sequence number is assigned (originated by the destination) to each route so that it can be known how old the route is. In routing table, whenever a route update is received, it is compared with existing route entry using sequence number. The route entry which has higher sequence number is maintained and other one is discarded. In case of different routes with same sequence number, the route with better metric is used. Updating of routing table may be periodic or immediate. Updation of Routing information in routing table can be performed in two ways. The first one is "full dump" in which node transmits the complete routing table. Second one is "incremental update" in which a node sends only those entries that have changed since last update in routing information. To avoid fluctuation in routing update, DSDV uses "settling time" to predict the time when route becomes stable. 
Table1. DSDV parameter and its default value

\begin{tabular}{|l|c|c|}
\hline \multicolumn{1}{|c|}{ Parameter } & $\begin{array}{c}\text { Default } \\
\text { Value }\end{array}$ & Range \\
\hline MAX_QUEUE_LENGTH & 5 & 1 to 10 \\
\hline DSDV_STARTUP_JITTER & 2.0 & 1 to 10 \\
\hline DSDV_ALMOST_NOW & 0.1 & 0.1 to 1 \\
\hline DSDV_BROADCAST_JITTER & 0.01 & $\begin{array}{c}0.001 \text { to } \\
1\end{array}$ \\
\hline DSDV_MIN_TUP_PERIOD & 1.0 & 1 to 10 \\
\hline IP_DEF_TTL & 32 & 1 to 50 \\
\hline
\end{tabular}

Despite all properties discussed above, there is variability in the performance of DSDV which is controlled by DSDV's control parameters significantly. Further, finding the optimal values for parameters configuration and understanding their impact on protocol performance is difficult task. Table 1 shows the main parameters with default values and its range [9]. It is evident from the table there would be more than $2^{29}$ possible combinations of parameters. Testing each set of parameter configuration in ns-2 is unfeasible. This situation motivates us to use some metaheuristic to solve this combinatorial optimization problem. There are huge combinations of parameters configuration values, only some sets of parameters are shown in Table2. These sets are called search space or solution vectors. In order to compare default and optimized DSDV parameter configuration, we have measured the resulted network performance (QoS) by considering following three metrics.

Packet Delivery Ratio (PDR): This is ratio of the number of packet received complete and correct by the destination and number of packets originated by the source.

Average End to End delay (AEED): This is average time consumed by data packet in travelling from source to destination.

Normalized Routing Load (NRL): It is the average number of routing packets that are transmitted for delivery of one data packet.

Table 2. Search Space(Combination of Values of Parameters)

\begin{tabular}{|c|c|c|c|c|c|c|}
\hline $\begin{array}{c}\text { Seri } \\
\text { al } \\
\text { No. }\end{array}$ & $\mathbf{M}_{\mathbf{L}} \mathbf{Q}_{-}$ & D_S & $\underset{\mathbf{N}}{\mathbf{D}_{\mathbf{N}} \mathbf{A}_{-}}$ & $\underset{\_J}{\text { D_B }}$ & $\underset{-\mathbf{P}}{\text { D_M_T }}$ & $\underset{\mathbf{T}}{\mathbf{I} \_\mathbf{D}}$ \\
\hline 1 & 3 & 2 & 0.7 & 0.03 & 1 & 43 \\
\hline 2 & 5 & 9 & 1 & 0.06 & 10 & 5 \\
\hline 3 & 8 & 7 & 0.4 & 0.08 & 4 & 22 \\
\hline 4 & 6 & 8 & 0.6 & 0.05 & 2 & 16 \\
\hline 5 & 2 & 4 & 0.3 & 0.06 & 6 & 38 \\
\hline 6 & 9 & 6 & 0.9 & 0.02 & 7 & 41 \\
\hline 7 & 5 & 4 & 0.6 & 0.05 & 5 & 33 \\
\hline 8 & 4 & 1 & 0.8 & 0.07 & 3 & 18 \\
\hline 9 & 8 & 6 & 0.2 & 0.04 & 8 & 26 \\
\hline 10 & 2 & 4 & 0.5 & 0.09 & 5 & 32 \\
\hline $\begin{array}{l}\text { M_Q } \\
\text { D_B } \\
\text { D_S } \\
\text { D_M } \\
\text { D_A } \\
\text { I_D_ }\end{array}$ & $\begin{aligned} &=\mathrm{NAX} \\
&= \mathrm{DSDV} \\
&= \mathrm{DSDV} \\
& \Gamma \_\mathrm{P}=\mathrm{D} \\
& \mathrm{J}=\mathrm{DSD} \\
&=\text { IP_DE }\end{aligned}$ & $\begin{array}{l}\text { QUEU } \\
\text { BROA } \\
\text { STAR' } \\
\text { V_M } \\
\text { ALM } \\
\text { TTL }\end{array}$ & $\begin{array}{l}\text { LENG } \\
\text { CAST_ } \\
\text { IP_JITT } \\
\text { TUP_L } \\
\text { T_NO }\end{array}$ & $\begin{array}{l}\text { CTER } \\
\mathrm{R} \\
\text { RIOD }\end{array}$ & & \\
\hline
\end{tabular}

\section{FRAMEWORK OF OPTIMIZATION}

Our framework of optimization consists of two parts one is an optimization algorithm and other is solution evaluation as shown in Figure 1. In optimization algorithm, 10 sets of solution vector (also called new population) is generated by PSO and used in simulation for performance evaluation. With the help of fitness function in equation (1) [6], PSO tries to find out an optimal parameters configuration in the search space (solution vector). After receiving a population from PSO, the simulation model passes one by one solution (values of parameters configuration) into DSDV. Now ns-2 takes VANET realistic instance, simulation parameters as shown in Table 3 and configures accordingly.

We have modified ns-2 in such a manner so that it can accept population from PSO automatically. After simulation, ns-2 produces global information known as simulation trace. With this information we calculate Packet Delivery Ratio (PDR), Average End-to-End Delay (AEED) and Normalized routing Load (NRL) of the whole mobile scenario. These three metric are used in calculation of the fitness function as follows [6].

$$
\text { Fitness }=w_{1} N R L+w_{2} A E E D-w_{3} P D R
$$

From the equation it can be seen that PDR is used with negative sign so that PDR gets priority over others. The purpose of this equation is to maximize PDR and other two NRL and AEED is to be minimized. This equation is aggregate minimization function and weights $\mathrm{w}_{1}, \mathrm{w}_{2}$, and $\mathrm{w}_{3}$ $(0.3,0.2$ and 0.5 respectively) are used to weigh the effect of each QoS parameters on the fitness value.

Table 3. Simulation Parameters for VANET

\begin{tabular}{|l|l|}
\hline \multicolumn{1}{|c|}{ Parameters } & \multicolumn{1}{c|}{ Value } \\
\hline Simulation time & 3 Minutes \\
\hline Simulation area & $1000 X 1000 \mathrm{~m}^{2}$ \\
\hline Number of vehicles & 30 Vehicles \\
\hline Vehicle speed & $0-50 \mathrm{~km} / \mathrm{hr}$ \\
\hline Propagation model & Two Ray Ground \\
\hline Radio frequency & $2.47 \mathrm{GHz}$ \\
\hline Channel bandwidth & $2 \mathrm{Mbps}$ \\
\hline Mac protocol & Mac/802_11 \\
\hline Transmission range of vehicles & $250 \mathrm{~m}$ \\
\hline CBR data flow & $15 \mathrm{Sessions}$ \\
\hline
\end{tabular}




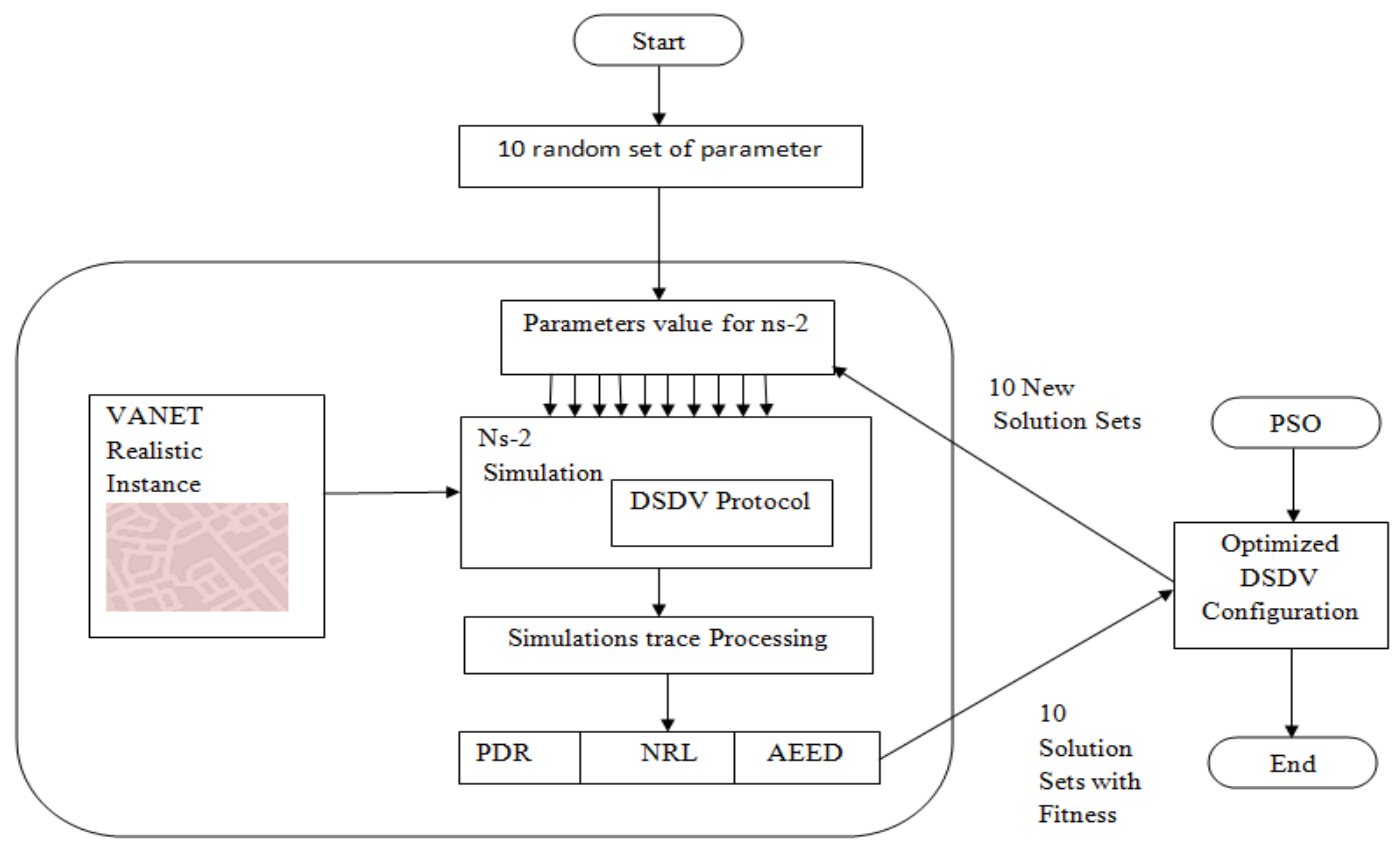

Figure 1: The optimization framework DSDV using PSO. PSO invokes the ns-2 simulator for each solution evaluation.

\section{VANET SCENARIO AND MOBILITY MODEL}

Since generation of an actual VANET scenario needs a large number of resources and people, we have used traffic/ network simulator to generate movement of vehicles as well as communication activity. With the help of digital map available [9] we have generated a realistic VANET environment. In this map we have considered actual road direction, signal lights, and traffic rules.

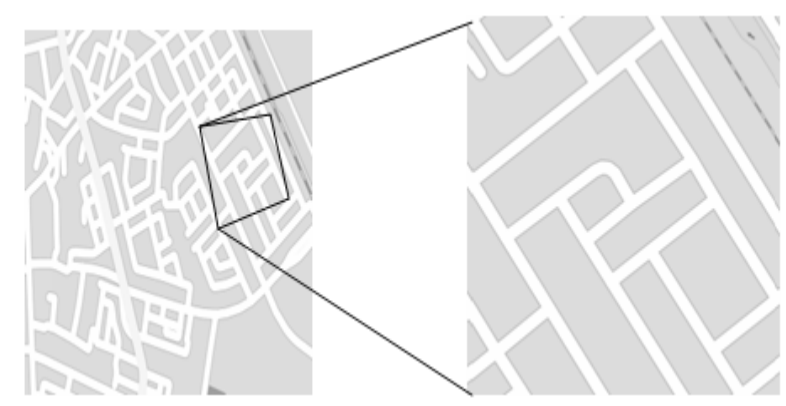

Figure. 2. Ghaziabad [9] Real VANET Scenarios. The maximum selected Area is $1000 \times 1000 \mathrm{~m}^{2}$.

For the simulation we selected a real map of Ghaziabad UP India $\left(1000 \times 1000 \mathrm{~m}^{2}\right)$ using Java OpenStreetMap editor (JOSM) [10]. Then map is converted into SUMO supported format with the help of netconvert [11]. The map of the area referenced in simulation is shown in Figure 2. On this map we have generated traffic in terms of number of vehicles, connection, turn and flows. The output of SUMO simulator is tcl script, which works as input for ns-2 simulator. In this simulation CBR is used as network application and UDP as source agent in vehicles. The packet size of CBR is 1000 bytes and bit rate is $64 \mathrm{~kb}$ per second. In this simulation we performed 15 sessions.

\section{EXPERIMENTAL SETUP}

The PSO algorithm is implemented in C++. To integrate PSO with ns-2, we have used shell programming. For experiment, we have made 50 independent run of PSO on machine with Pentium IV, 2.4 GHz core, 2Gb RAM, and Linux Ubuntu 12.05 LTS. For the generalization of the result each independent run performed different communication pattern and vehicular mobility.

\subsection{Problem mapping into PSO} PSO Algorithm [11]

Input: 10 sets of 6 parameters value

Output: Optimized 10 sets of 6 parameters value

Begin:

1. Initialize a population array of particles with random positions and velocities on $\mathrm{D}$ dimensions in the search space.

2. loop

a. For each particle, evaluate the desired optimization fitness function in $\mathrm{D}$ variables.

b. Compare particle's fitness evaluation with its pbest $_{\mathrm{i}}$. If current value is better than pbest $\mathrm{i}_{\mathrm{i}}$, then set pbest $t_{\mathrm{i}}$ equal to the current value, and $\mathrm{p}_{\mathrm{i}}$ equal to the current location $\mathrm{x}_{\mathrm{i}}$ in the $\mathrm{D}$ dimensional space.

c. Identify the particle in the neighborhood with the best success so far, and assign its index to the variable $\mathrm{g}$. 
d. Change the velocity and position of the particle according to the equations in 2 and 3 .

$$
\begin{aligned}
\mathrm{v}_{\mathrm{i}}= & \mathrm{v}_{\mathrm{i}}+\mathrm{U}\left(0, \phi_{1}\right) \otimes\left(\mathrm{p}_{\mathrm{i}}-\mathrm{x}_{\mathrm{i}}\right)+\mathrm{U}\left(0, \phi_{2}\right) \otimes \\
& \left(\mathrm{p}_{\mathrm{g}}-\mathrm{x}_{\mathrm{i}}\right) \\
\mathrm{x}_{\mathrm{i}}= & \mathrm{x}_{\mathrm{i}}+\mathrm{v}_{\mathrm{i}}
\end{aligned}
$$

e. If a criterion is met, exit loop.

3. end loop

End

\section{Modified PSO according to our Problem}

Begin

1. Initialize the population as 10 random sets of 6 parameters. Here search dimension is 6 . Where $i=1$, $2,3 \ldots 10$.

2. Loop

a. Value of the fitness function of 10 sets $\left(\mathrm{x}_{\mathrm{i}}\right)$ is evaluated by $\mathrm{ns}-2$ in the form of communication cost $\left(\mathrm{c}_{\mathrm{i}}\right)$ as mentioned in figure 1 . Where $i=1,2,3, \ldots 10$.

b. In first generation, value of pbest $\mathrm{i}_{\mathrm{i}}$ is equal to communication cost $\left(c_{i}\right)$ and $p_{i}$ is set to $\mathrm{x}_{\mathrm{i}}$. The $\mathrm{x}_{\mathrm{i}}$ denotes the current position of $i^{\text {th }}$ set in 6 dimension search space. For successive generations the fitness value $c_{i}$ for $\mathrm{x}_{\mathrm{i}}$ is compared with pbest $\mathrm{t}_{\mathrm{i}}$. If $\mathrm{c}_{\mathrm{i}}$ is better than pbest $\mathrm{i}_{\mathrm{i}}$, set pbest $\mathrm{i}_{\mathrm{i}}$ equal to $\mathrm{c}_{\mathrm{i}}$ and $\mathrm{p}_{\mathrm{i}}$ equal to the current location $\mathrm{x}_{\mathrm{i}}$ in the 6ndimentional space. Where $\mathrm{i}=1,2$, $3, \ldots 10$.

c. The value of $\mathrm{i}$ corresponding to minimum pbest $_{\mathrm{i}}$ is selected and assigned to $\mathrm{g}$.

d. The initial velocity of first generation is set to zero. The successive velocity and position are calculated according to equation 2 and 3 respectively. The value of $\phi_{1}$ and $\phi_{2}$ is set equal to 2 . The symbol $\otimes$ denotes the component wise multiplication. $\mathrm{U}\left(0, \phi_{\mathrm{k}}\right)$ represents a random number between 0 and $\phi_{\mathrm{k}}$. Where $\mathrm{k}=1,2$.

e. Here condition is checked to terminate the loop after evaluating 50 successive generations.

3. The loop terminates after successful evaluation of 50 generations.

End

\subsection{Result Analysis}

Above experiment is conducted for 10 different seed values on the map. PSO has been performed for 50 iteration or generation and it gives a solution corresponding to each seed. Now, out of 10 solutions on different seed, we have taken best solution along with default parameters of DSDV which is shown in table 4.

Table 4. Optimized and default value of parameters

\begin{tabular}{|l|c|c|}
\hline \multicolumn{1}{|c|}{ Parameter } & $\begin{array}{c}\text { Default } \\
\text { Value }\end{array}$ & $\begin{array}{c}\text { Optimal } \\
\text { value }\end{array}$ \\
\hline MAX_QUEUE_LENGTH & 5 & 3 \\
\hline DSDV_STARTUP_JITTER & 2.0 & 1 \\
\hline DSDV_ALMOST_NOW & 0.1 & 1 \\
\hline DSDV_BROADCAST_JITTER & 0.01 & 1 \\
\hline DSDV_MIN_TUP_PERIOD & 1.0 & 3 \\
\hline IP_DEF_TTL & 32 & 18 \\
\hline
\end{tabular}

A comparative analysis of different QoS parameters is shown in Table 5. It is evident from the table that there is $5.74 \%$ increment in PDR, $11.96 \%$ drop in AEED, $5.42 \%$ drop in NRL and $11.69 \%$ drop in communication cost.

Table 5. Comparative analysis of different QoS parameters

\begin{tabular}{|l|l|l|l|}
\hline QoS Parameter & Default & Optimized & \% Change \\
\hline PDR & 0.547632 & 0.579062 & 05.74 \\
\hline AEED & 0.096286 & 0.084763 & 11.96 \\
\hline NRL & 2.532919 & 2.396080 & 05.42 \\
\hline $\begin{array}{l}\text { Communication } \\
\text { Cost }\end{array}$ & 0.505317 & 0.446246 & 11.69 \\
\hline
\end{tabular}

\section{CONCLUSIONS \& FUTURE WORK}

DSDV is proactive routing protocol and the performance of the protocol in given scenario depends on the parameters configuration as discussed above. Combinations of parameters configuration are multiples and finding optimal set of parameters configuration by testing each set on real scenario is not practical. Using meta-heuristic in such problem which has combinatorial nature is better. Therefore in this paper, we have obtained optimal value of parameter configuration of DSDV, using PSO (a meta-heuristic). These optimal values help in minimization of communication cost and improve the QoS. The use of PSO has shown measurable improvement in QoS of DSDV. There is $11.96 \%, 5.42 \%$ and $11.69 \%$ drops in AEED, NRL and communication cost respectively. There is $5.74 \%$ gain in PDR. Since, one of the factors for performance of a routing protocol depends on its parameters configuration. Therefore, in future work we will try to use some other metaheuristic technique on DSDV or on some other protocol like UDP, DSR etc. to optimize them for given scenario. 


\section{REFERENCES}

[1] H"arri, J., Filali, F., Bonnet, C.: Mobility Models for Vehicular Ad Hoc Networks: A Survey and Taxonomy. Research Report RR-06-168 (March 2007).

[2] Blum, C., Roli, A.: Metaheuristics in combinatorial optimization: Overview and conceptual comparison. ACM Computing Surveys 35(3), 268-308 (2003)

[3] E. Alba, B. Dorronsoro, F. Luna, A. Nebro, P. Bouvry, and L. Hogie, "A Cellular MOGA for Optimal Broadcasting Strategy in Metropolitan MANETs," Computer Communications , vol. 30, no. 4, pp. 685 697, (2007)

[4] Vanhatupa, T., H"annik"ainen, M., H"am"al"ainen, T.: Optimization of mesh WLAN channel assignment with a configurable genetic algorithm. In: WiMeshNets 2006 (2006)

[5] Di Caro, G.A., Ducatelle, F., Gambardella, L.M.: AntHocNet: An Adaptive Nature-Inspired Algorithm for Routing in Mobile Ad Hoc Networks. European Transactions on Telecommunications 16(5), 443-455 (2005)
[6] Garc'a-Nieto, J. Toutouh, and E. Alba, "Automatic Parameter Tunning with Metaheuristics of the AODV Routing Protocol for Vehicular Ad-hoc Networks," EvoApplications, part II. LNCS 6025, pp. 21-30, 2010.

[7] Huang, C., Chuang, Y., Hu, K.: Using particle swarm optimization for QoS in ad-hoc multicast. Eng. Appl. of Artificial Intelligence (2009) (in Press)

[8] Perkins, C.E., Bhagwat, P.: Highly Dynamic DestinationSequenced Distance-Vector Routing (DSDV) for Mobile Computers. In: ACM SIGCOMM 1994, London, UK, pp. 234-244 (1994)

[9] OOpenStreetMapcontributors(www.openstreetmap.org/c opyright).

[10] Java OpenStreetMap editor," [online] http:// josm. openstreetmap.de

[11] Poli, Riccardo, James Kennedy, and Tim Blackwell, "Particle swarm optimization" Swarm intelligence 1.1 pp. 33-57, 2007 